\title{
ANALYSIS OF VAN DER WAAL EQUATION NEAR THE CRITICAL POINT
}

\author{
Awuor, J.B.
}

Department of Physics, University of Nairobi, P.O. Box 30197, 00100 Nairobi

\begin{abstract}
The van der Waal equation of state is redefined in a functional manner that reveals some additional critical parameters and presents the ideal gas temperature as a lower limit to the van der Waal gas temperature. The law of corresponding states is also shown to apply to the lower limits of reduced temperatures.
\end{abstract}

\section{INTRODUCTION}

An equation of state for a given gas system can be determined in terms of pressure, volume, temperature and mass of the enclosed gas under consideration. At sufficiently low pressures, the relations among these quantities are found to satisfy the empirical ideal gas law, that is

$$
P_{m} V_{m}=N_{m} k_{m} T_{m}
$$

where $P_{m}, V_{m}, T_{m}, N_{m}$ and $k_{m}$ are pressure, volume, temperature, number of particles and Boltzmann constant respectively, while the subscript $m$ is introduced to specify that the parameters are determined or deduced from experimental measurements. At relatively higher pressures, measurements are found to relate in manners that deviate from that of Eqn (1). In an attempt to account for such deviations, van der Waal took into account the effects of intermolecular forces ${ }^{1,2}$ and theoretically developed general equation of state for gases as ${ }^{3,4,5}$

$$
\left[P_{m}+\frac{\alpha N_{m}^{2}}{V_{m}^{2}}\right]\left[V_{m}-N_{m} b\right]=N_{t} k_{t} T_{t}
$$

where $\alpha$ is a factor dependent on the strength of the attractive intermolecular forces and $b$ is a factor dependent on molecular sizes and the repulsive intermolecular forces, while the subscript $t$ is introduced to indicate that the quantities $T_{t}, N_{t}, k_{t}$ are theoretically implied values of temperature, number of particles and Boltzmann constant respectively. It has been experimentally verified that the parameters $\alpha$ and $b$ are constants, that are characteristic of the system under consideration. For any particular gas system, the van der Waal equation becomes identical to the ideal gas law in the limit when the volume $V$ is large.

The format of Eqn (2) is often interpreted to imply that in the determination of $T_{t}$, the parameters $P_{m}$ and $V_{m}$ are independent of $\alpha$ and $b$. We shall show that this assumption does not hold in the neighborhood of a critical point. In addition we shall highlight the relation between the empirical ideal gas temperature $T_{m}$ and the theoretically implied van der Waal temperature $T_{t}$.

\section{METHOD}

\section{Basic formulations}

We consider here that, the intermolecular forces considered in the van der Waal equation are already in play so that the results obtained from measurements of pressure, volume and temperature for real gases, can be represented by the functional forms $P(\alpha, b), V(b)$ and $T(\alpha, b, k)$, so that Eqn (1) becomes

$$
P_{m}(\alpha, b) V_{m}(b)=N_{m} k_{m} T_{m}\left(\alpha, b, k_{m}\right)
$$

Next we seek a form of van der Waal equation, analogous to Eqn (3), in which the characteristic quantities $\alpha$ and $b$ do not appear explicitly. To achieve this we rewrite Eqn (2) in the form

$$
P_{t}(\alpha, b) V_{t}(b)=N_{t} k_{t} T_{t}\left(\alpha, b, k_{t}\right)
$$


where we have made the replacements

$$
P_{t}(\alpha, b)=P_{m}(\alpha, b)+\frac{\alpha N_{m}^{2}}{V_{m}^{2}(b)}
$$

and

$$
V_{t}(b)=V_{m}(b)-N_{m} b
$$

Equation (4) is a representation of the van der Waal equation of state in a manner that preserves the format of the ideal gas law. Thus for any volume $V_{m}$, we may combine Eqns (4), (5) and (6) to obtain

$$
\left[P_{m}(\alpha, b)+\frac{\alpha N_{m}^{2}}{V_{m}^{2}(b)}\right]\left[V_{m}(b)-N_{m} b\right]=N_{t} k_{t} T_{t}(\alpha, b, k)
$$

which can be rearranged to become

$$
\begin{aligned}
& V_{m}^{3}(b)-\left[N_{m} b+\frac{N_{t} k_{t} T_{t}(\alpha, b, k)}{P_{m}(\alpha, b)}\right] \\
& V_{m}^{2}(b)+\frac{\alpha N_{m}^{2}}{P_{m}(\alpha, b)} V_{m}(b)-\frac{\alpha N_{m}^{3} b}{P_{m}(\alpha, b)}=0
\end{aligned}
$$

This is a cubic function $V_{m c}(b)$ in and would in general have three solutions. Graphically, the special condition when all the three solutions are equal describes a point of inflection which is called the critical point and for which we introduce subscript $c$ hereafter. Thus in the neighborhood of a critical point, Eqn (8) becomes

$$
\begin{aligned}
& V_{m c}^{3}(b)-\left[N_{m c} b+\frac{N_{t c} k_{t c} T_{t c}\left(\alpha, b, k_{t c}\right)}{P_{m c}(\alpha, b)}\right] \\
& V_{m c}^{2}(b)+\frac{\alpha N_{m c}^{2}}{P_{m c}(\alpha, b)} V_{m c}(b)-\frac{\alpha N_{m c}^{3} b}{P_{m c}(\alpha, b)}=0
\end{aligned}
$$

Since the cubic polynomial

$$
\begin{aligned}
& V_{m c}^{3}(b)-3 V_{m c}(b) V_{m c}^{2}(b)+ \\
& 3 V_{m c}^{2}(b) V_{m c}(b)-V_{m c}^{3}(b)=0
\end{aligned}
$$

also has three equal solutions, we consequently compare its coefficients ${ }^{1,6}$ with those of Eqn (9) to obtain the critical parameters as

$$
V_{m c}(b)=3 N_{m c} b
$$

$$
P_{m c}(\alpha, b)=\frac{\alpha}{27 b^{2}}
$$

and

$T_{t c}\left(\alpha, b, k_{t c}\right)=\frac{8 \alpha N_{m c}}{27 b k_{t c} N_{t c}}$

from which we obtain the well established relation ${ }^{7}$

$$
\frac{P_{m c}(\alpha, b) V_{m c}(b)}{N_{t c} k_{t c} T_{t c}\left(\alpha, b, k_{t c}\right)}=\frac{3}{8}
$$

\section{Additional Critical Parameters}

On substituting Eqns (11a), (11b) and (11c) into Eqns (3), (4) and (6) we obtain the following additional critical parameters

$$
\begin{aligned}
& V_{t c}(b)=2 N_{m c} b, \\
& P_{t c}(\alpha, b)=\frac{4 \alpha}{27 b^{2}},
\end{aligned}
$$

and

$$
T_{m c}\left(\alpha, b, k_{m c}\right)=\frac{\alpha}{9 b k_{m c}}
$$

By substituting Eqn (13c) into (11c) we obtain the relation

$$
T_{t c}\left(\alpha, b, k_{t c}\right)=\frac{8}{3}\left[\frac{N_{m c} K_{m c}}{N_{t} K_{t}}\right] \frac{T_{m}\left(\alpha, b, k_{m}\right)}{}
$$

from which it is clearly evident that if the temperatures $T_{m}$ and $T_{t}$ are equal, then the number of enclosed particles that do participate at the critical point must be such that $N_{m c} \leq N_{t c}$, that is, provided that the Boltzmann parameter is a universal constant.

If we consider that Eqn (14) applies at points sufficiently removed from the critical point, then we may write 
$T_{t}\left(\alpha, b, k_{t}\right)=\frac{8}{3}\left[\frac{N_{m} K_{m}}{N_{t} K_{t}}\right] \frac{T_{m}\left(\alpha, b, k_{m}\right)}{\underline{n^{\prime}}}$

as a general relation between the van der Waal's theoretical temperature $T_{t}$ and the empirically determined temperature $T_{m}$. Eqn (7) therefore becomes

$$
\begin{aligned}
& {\left[P_{m}(\alpha, b)+\frac{\alpha N_{m}^{2}}{V_{m}^{2}(b)}\right]\left[V_{m}(b)-N_{m} b\right]} \\
& =\frac{8}{3} N_{m} k_{m} T_{m}\left(\alpha, b, k_{m}\right)
\end{aligned}
$$

This is a form of van der Waal equation of state in which the pressure, volume and temperature are all experimentally measured values.

\section{The Law of Corresponding States}

If we introduce a dimensionless parameter notation

$$
\bar{X}=\frac{X}{X_{c}},
$$

where $X_{c}$ is the critical value for any $X$ (representing any specific parameter amongst pressure, volume, temperature, number of particles and Boltzmann constant), then we have for example,

$$
\begin{aligned}
& \bar{V}_{m}(b)=\frac{V_{m}(b)}{V_{m c}(b)}, \\
& \bar{P}_{m}(\alpha, b)=\frac{P_{m}(\alpha, b)}{P_{m c}(\alpha, b)}
\end{aligned}
$$

and

$$
\bar{T}_{m}\left(\alpha, b, k_{m}\right)=\frac{T_{m}\left(\alpha, b, k_{m}\right)}{T_{m c}\left(\alpha, b, k_{m c}\right)} .
$$

Taking Boltzmann constant as a universal constant, we therefore set

$$
k_{m}=k_{m c}
$$

so that on substituting eqns (17b), (17c), (17d) and (13c) into Eqn (16) we obtain

$$
\left(\bar{P}_{m}(\alpha, b)+\frac{3}{\bar{V}_{m}^{2}(b)}\right)\left(\bar{V}_{m}(b)-\frac{1}{3}\right)=\frac{8}{3} \bar{N}_{m} \bar{T}_{m}\left(\alpha, b, k_{m}\right)
$$

as a reduced equation of state with all parameters obtained from practical measurements.

By dividing Eqn (15) by (14) we obtain the reduced relation

$\bar{T}_{t}\left(\alpha, b, k_{t}\right)=\left[\frac{\bar{N}_{m} \bar{k}_{m}}{\bar{N}_{t} \bar{k}_{t}}\right] \frac{\bar{T}_{m}\left(\alpha, b, k_{m}\right)}{}$

Since in the formulation of van der Waal equation of state, a theoretical assumption is made that all particles do participate at the critical point, such that

$\bar{N}_{t}=1$,

we can by making use of Eqns (18), (20) and (21), reduce Eqn (19) to the form

$$
\left(\bar{P}_{m}(\alpha, b)+\frac{3}{\bar{V}_{m}^{2}(b)}\right)\left(\bar{V}_{m}(b)-\frac{1}{3}\right)=\frac{8}{3} \bar{T}_{t}\left(\alpha, b, k_{t}\right)
$$

which is the familiar law of corresponding states ${ }^{6,8,9,10}$. Eqn (22) is applicable universally to any form of van der Waal gas, because the quantities and no longer appear explicitly. This universality has been experimentally confirmed ${ }^{11,12}$ for many substances. Any two different substances for which the reduced parameters, $\bar{X}$ are equal, are said to be in corresponding states.

\section{DISCUSSIONS}

Although the universality tests for the law of corresponding states have been based on Eqn (22), with the assumption that $\bar{T}_{t}=\bar{T}_{m}$, it is clear from Eqn (19) that the temperatures need not always be equal. From statistical arguments, not all particles may simultaneously participate at the critical point. This implies the condition 


$$
\bar{N}_{m} \geq 1
$$

and consequently that

$\bar{T}_{t}\left(\alpha, b, k_{t}\right) \geq \bar{T}_{m}\left(\alpha, b, k_{m}\right)$

Thus the ideal gas temperature provides the lower limit to the van der Waal gas temperature.

In addition, we note that in the reduced form, the difference between the temperatures $\bar{T}_{m}$ and $\bar{T}_{t}$ is minimal, compared to that between $T_{m}$ and $T_{t}$. The minimization of the deference consequently renders the law of corresponding states to be in far more exact agreement with experimental results than the van der Waals equation. For very dilute gases, where we can neglect the terms involving $\alpha$ and $b$, the reduced temperatures $\bar{T}_{m}$ and $\bar{T}_{t}$ become nontrivially equal.

Finally, we note that without the definitions introduced in Eqns (4), (5) and (6), the van der Waal equation of state can equivalently be expressed in the form

$$
\left[P_{m}(0,0)+\frac{\alpha N_{m}^{2}}{V_{m}^{2}(0)}\right]\left[V_{m}(0)-N_{m} b\right]=N_{t} k_{t} T_{t}\left(\alpha, b, k_{t}\right)
$$

so that the ideal gas law consequently becomes

$$
\left[P_{t}(0,0)\right]\left[V_{t}(0)\right]=N_{t} k_{t} T_{t}\left(0,0, k_{t}\right)
$$

Although Eqn (25) fits the usual interpretations of van der Waal equation, the details introduced here imply that this formulation is however inconsistent with concept of critical parameters, unless the parameters are themselves independent of $\alpha$ and $b$, such that $P_{m c}(0,0)=P_{m c}(\alpha, b)$ and $V_{m c}(0)=V_{m c}(b)$. This would however lead to an erroneous conclusion that the effects of intermolecular forces are not measurable.

\section{REFERENCES}

1. Kerson Huang, Introduction to Statistical Physics, Taylor and Francis, London (2001) p.49-55.

2. Sears F.W. and Salinger G.L., Thermodynamics, Kinetic Theory and Statistical Thermodynamics, 3rd Ed. Addison-Wesley, Massachusetts (1975) p. 28, 276-278.

3. Rief F., Fundamentals of Statistical Thermal Physics, McGraw Hill, New York (1965) p.173.

4. Agarwal, B.K., and Eisner M., Statistical Mechanics, Wiley Eastern Ltd, New Delhi (1988) p.55, 118.

5. Gupta, M.C., Statistical Thermodynamics, Wiley Eastern Ltd, New Delhi (1990) p. 288-291.

6. Kerson Huang, Statistical Mechanics, Wiley Eastern Ltd, New Delhi (1963) p.40-46.

7. Rossini F.D., Thermodynamics and Physics of Matter, Oxford Univ. Press, London (1955) p. 434-437.

8. Rumer, Yu. B., and Ryvkin, M.Sh., Thermodynamics, Statistical Physics and kinetics, Mir Publishers, Moscow (1980) p.52-57, 334.

9. Sears, F.W., (1975) op cit, p. 50-51

10. Landau, L.D. and Lifshitz, E.M., Statistical Physics 2nd Revised Ed., Pergamon Press, Oxford (1969) p.270-272.

11. Guggenheim, E.A., J.Chem. Phys. 13, 253 (1945).

12. Rossini F.D., (1955) op cit, p. 422. 